\title{
Interaction-induced quantum ratchet in a Bose-Einstein condensate
}

\author{
Dario Poletti, ${ }^{1}$ Giuliano Benenti, ${ }^{2,3}$ Giulio Casati, ${ }^{2,3,1}$ and Baowen $\mathrm{Li}^{1,4,5}$ \\ ${ }^{1}$ Department of Physics and Centre for Computational Science and Engineering, National University of Singapore, \\ Singapore 117542, Republic of Singapore \\ ${ }^{2}$ CNISM, CNR-INFM and Center for Nonlinear and Complex Systems, Università degli Studi dell'Insubria, Via Valleggio 11, \\ 22100 Como, Italy \\ ${ }^{3}$ Istituto Nazionale di Fisica Nucleare, Sezione di Milano, via Celoria 16, 20133 Milano. Italy \\ ${ }^{4}$ Laboratory of Modern Acoustics and Institute of Acoustics, Nanjing University, 210093, People's Republic of China \\ ${ }^{5}$ NUS Graduate School for Integrative Sciences and Engineering, 117597, Republic of Singapore \\ (Received 21 September 2006; revised manuscript received 6 April 2007; published 24 August 2007)
}

\begin{abstract}
We study the dynamics of a dilute Bose-Einstein condensate confined in a toroidal trap and exposed to a pair of periodically flashed optical lattices. We first prove that in the noninteracting case this system can present a quantum symmetry which forbids the ratchet effect classically expected. We then show how many-body atom-atom interactions, treated within the mean-field approximation, can break this quantum symmetry, thus generating directed transport.
\end{abstract}

DOI: 10.1103/PhysRevA.76.023421 PACS number(s): 42.50.Vk, 05.60.-k, 03.75.Kk, 05.45.-a

The ratchet effect, that is, the possibility to drive directed transport with the help of zero-mean perturbations, has recently gained renewed attention due to its possible relevance for biological transport, molecular motors, and the prospects of nanotechnology [1,2]. At the classical level, the ratchet effect can be found in periodic systems due to a broken space-time symmetry [3]. The ratchet phenomenon has also been discussed in quantum systems [4], including the Hamiltonian limit without dissipation [5]. Experimental implementations of directed transport range from semiconductor heterostructures to quantum dots, Josephson junctions, and cold atoms in optical lattices [6].

Quantum Hamiltonian ratchets are relevant in systems such as cold atoms in which the high degree of quantum control may allow experimental implementations near the dissipationless limit. Moreover, the realization of BoseEinstein condensates (BECs) of dilute gases has opened new opportunities for the study of dynamical systems in the presence of many-body interactions. Indeed, it is possible to prepare initial states with high precision and to tune over a wide range the many-body atom-atom interaction. From the viewpoint of directed transport, the study of many-body quantum system is, to our knowledge, at the very beginning.

In this paper, we investigate the quantum dynamics of a BEC in a pair of periodically flashed optical lattices. We show how the interaction between atoms in the condensate, studied in the mean-field approximation, can break the quantum symmetry present in our model in the noninteracting limit, thus giving rise to the ratchet effect. The role of noise, the validity of the mean-field description, and the possibility to observe experimentally our ratchet model are discussed as well.

We consider $N$ condensed atoms confined in a toroidal trap of radius $R$ and cross section $\pi r^{2}$, with the condition $r$ $\ll R$, so that the motion is essentially one dimensional. The dynamics of a dilute condensate in a pair of periodically kicked optical lattices at zero temperature is described by the Gross-Pitaevskii nonlinear equation,

$$
i \frac{\partial}{\partial t} \psi(\theta, t)=\left[-\frac{1}{2} \frac{\partial^{2}}{\partial \theta^{2}}+g|\psi(\theta, t)|^{2}+V(\theta, \phi, t)\right] \psi(\theta, t),
$$

where $\theta$ is the azimuthal angle, $g=8 \mathrm{NaR} / r^{2}$ is the scaled strength of the nonlinear interaction (we consider the repulsive case, i.e., $g>0$ ), $a$ is the $s$-wave scattering length for elastic atom-atom collisions. The kicked potential $V(\theta, \phi, t)$ is defined as

$$
\begin{gathered}
V(\theta, \phi, t)=\sum_{n}\left[V_{1}(\theta) \delta(t-n T)+V_{2}(\theta, \phi) \delta(t-n T-\xi)\right] \\
V_{1}(\theta)=k \cos \theta, \quad V_{2}(\theta, \phi)=k \cos (\theta-\phi)
\end{gathered}
$$

where $k$ is the kicking strength and $T$ is the period of the kicks. The parameters $\phi \in[0,2 \pi]$ and $\xi \in[0, T]$ are used to break the space and time symmetries, respectively. Note that we set $\hbar=1$ and that the length and the energy are measured in units of $R$ and $\hbar^{2} / m R^{2}$, with $m$ the atomic mass. The wave function normalization reads $\int_{0}^{2 \pi} d \theta|\psi(\theta, t)|^{2}=1$ and boundary conditions are periodic, $\psi(\theta+2 \pi, t)=\psi(\theta, t)$.

We first consider the noninteracting case $g=0$. Here, when $\phi \neq 0, \pi$ and $\xi \neq 0, T / 2$, space-time symmetries are broken and there is directed transport, both in the classical limit and, in general, in quantum mechanics [7]. However, if we take $T=6 \pi$ and $\xi=4 \pi$, then the quantum motion, independently of the kicking strength $k$, is periodic of period $2 T$.

In order to prove this periodicity, it is useful to write the initial wave function as $\psi(\theta, 0)=\sum_{n} A_{n} \exp (\operatorname{in} \theta)$, where $A_{n}$ $=\frac{1}{2 \pi} \int_{0}^{2 \pi} \psi(\theta, 0) \exp (-i n \theta)$. After free evolution up to time $t$, the wave function becomes $\psi(\theta, t)=\Sigma_{n} A_{n} \exp \left(-i \frac{n^{2}}{2} t+i n \theta\right)$. If $t=4 \pi$ we have $\psi(\theta, t)=\psi(\theta, 0)$ while, if $t=2 \pi$, we obtain $\psi(\theta, t)=\psi(\theta+\pi, 0)$. Using these relations we can easily see that the system is periodic with period $12 \pi$. Indeed,

$$
\psi\left(\theta, 4 \pi^{+}\right)=\exp \left[-i V_{1}(\theta)\right] \psi(\theta, 0)
$$




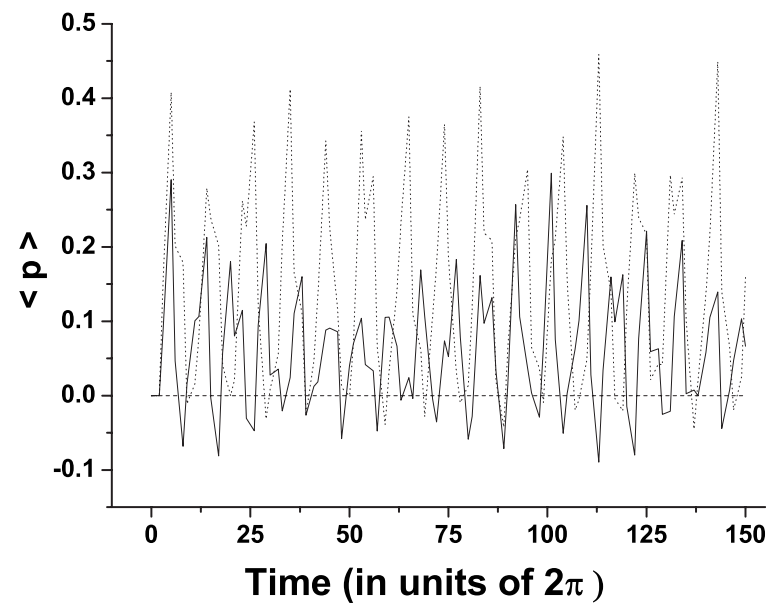

FIG. 1. Momentum vs time for different values of interaction strength $g$, at $k \approx 0.74$ and $\phi=-\pi / 4: g=0$ (dashed line), $g=0.5$ (continuous curve), $g=1$ (dotted curve).

$$
\begin{gathered}
\begin{aligned}
& \psi\left(\theta, 6 \pi^{+}\right)=\exp \left[-i V_{2}(\theta, \phi)\right] \psi\left(\theta+\pi, 4 \pi^{+}\right) \\
&=\exp \left\{-i\left[V_{2}(\theta, \phi)-V_{1}(\theta)\right]\right\} \psi(\theta+\pi, 0), \\
& \psi\left(\theta, 10 \pi^{+}\right)=\exp \left[-i V_{1}(\theta)\right] \psi\left(\theta, 6 \pi^{+}\right) \\
&=\exp \left[-i V_{2}(\theta, \phi)\right] \psi(\theta+\pi, 0), \\
& \psi\left(\theta, 12 \pi^{+}\right)=\exp \left[-i V_{2}(\theta, \phi)\right] \psi\left(\theta+\pi, 10 \pi^{+}\right)=\psi(\theta, 0),
\end{aligned}
\end{gathered}
$$

where $\psi\left(\theta, t^{+}\right)$denotes the value of the wave function at time $t$ just after the kick. The momentum $\langle p(t)\rangle$ $=-i \int_{0}^{2 \pi} d \theta \psi^{\star}(\theta, t) \frac{\partial}{\partial \theta} \psi(\theta, t)$ also changes periodically with period $12 \pi$ (four kicks). Therefore the average momentum $p_{\mathrm{av}}=\lim _{t \rightarrow \infty} \bar{p}(t)\left(\bar{p}(t)=\frac{1}{t} \int_{0}^{t} d t^{\prime}\left\langle p\left(t^{\prime}\right)\right\rangle\right)$ is given by

$$
\begin{aligned}
p_{\mathrm{av}} & =\frac{4 \pi\langle p(0)\rangle+2 \pi\left\langle p\left(4 \pi^{+}\right)\right\rangle+4 \pi\left\langle p\left(6 \pi^{+}\right)\right\rangle+2 \pi\left\langle p\left(10 \pi^{+}\right)\right\rangle}{12 \pi} \\
& =\langle p(0)\rangle+\frac{k}{2} \int_{0}^{2 \pi}[\sin (\theta)-\sin (\theta-\phi)]|\psi(\theta, 0)|^{2} d \theta
\end{aligned}
$$

In particular, for the constant initial condition $\psi(\theta, 0)$ $=1 / \sqrt{2 \pi}$, which is the ground state of a particle in the trap, the momentum remains zero at any later time. This initial condition has an important physical meaning, as it corresponds to the initial condition for a Bose-Einstein condensate.

It is therefore interesting to study the case of a BEC because atom-atom interactions may break the above periodicity, and this may cause generation of momentum. The numerical integration of Eq. (1) confirms this expectation: as shown in Fig. 1, at $g \neq 0$ the momentum oscillates around a mean value clearly different from zero. Notice that without interactions $(g=0)$ the momentum is exactly zero, so that directed transport is induced by the many-body atom-atom interactions.

In Fig. 2, we compare the asymptotic value $p_{\text {av }}$, obtained from long numerical integrations of the Gross-Pitaevskii

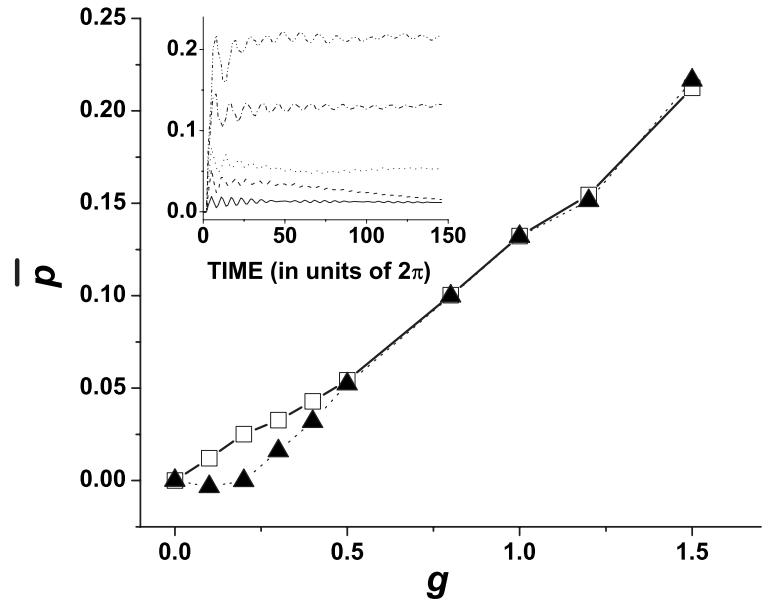

FIG. 2. Momentum averaged over the first 30 kicks (solid line with boxes) and asymptotic momentum (dotted line with triangles). Inset: Cumulative average $\bar{p}(t)$ as a function of time for different values of $g$. From bottom to top $g=0.1,0.2,0.4,1.0,1.5$. Parameter values: $k \approx 0.74, \phi=-\pi / 4$.

equation (dotted line with triangles), with the average of $\langle p(t)\rangle$ over the first 30 kicks $[\bar{p}(90 \pi)$, continuous line with boxes]. It can be seen that this short-time average is sufficient to obtain a good estimate of the average momentum $p_{\text {av }}$, provided that $g \geq 0.5$. It is interesting to remark that the average momentum after the first kicks grows monotonically with $g$. Therefore the ratchet current provides a method to measure the interaction strength in an experiment. In the inset of Fig. 2 we show the cumulative average $\bar{p}(t)$. For strong enough interactions $(g \gtrless 0.5)$ the convergence to the limiting value $p_{\mathrm{av}}$ is rather fast as we can already see from the main part of Fig. 2.

Since the above complete periodicity of the single particle system $(g=0)$ is a very fragile quantum phenomenon, it is important to check the visibility of the ratchet effect when the unavoidable noise leads to a departure from the ideal periodic behavior. For this purpose we consider fluctuations in the kicking period, modeled as random and memoryless variations of the period between consecutive kicks, with the fluctuation amplitude at each kick randomly drawn from a uniform distribution in the interval $[-\epsilon, \epsilon]$. We have seen that, when the size of the fluctuations is $\epsilon=T / 200$, then the ratchet current generated in the noninteracting case is $\bar{p}(90 \pi)=-0.007$. This value of $\bar{p}$ is much smaller than the genuine many-body ratchet current already shown in Fig. 2 for values of $g \gtrsim 0.2$. A similar conclusion is obtained when the kicks are substituted by more realistic Gaussian pulses of width $T / 10$. In this case $\bar{p}$ after 30 kicks is equal to $\bar{p}(90 \pi)=-0.01$, again too small to hide or to be confused with the many-body ratchet effect.

The interaction-induced generation of a nonzero current can be understood as follows. We approximate, for small values of $g$, the free evolution of the BEC by a split-operator method as in [8]:

$$
\psi(\theta, \tau) \approx e^{-i(1 / 2)\left(\partial^{2} / \partial \theta^{2}\right)(\tau / 2)} e^{-i g|\tilde{\psi}(\theta, \tau / 2)|^{2} \tau} e^{-i(1 / 2)\left(\partial^{2} / \partial \theta^{2}\right)(\tau / 2)} \psi(\theta, 0),
$$

where $\tilde{\psi}(\theta, t+\Delta t)=e^{-i(1 / 2)\left(\partial / \partial \theta^{2}\right) \Delta t} \psi(\theta, t)$. 
In particular, we obtain

$$
\psi(\theta, 4 \pi) \approx \exp \left[-i 4 \pi g|\psi(\theta, 0)|^{2}\right] \psi(\theta, 0)
$$

and

$$
\begin{aligned}
\psi(\theta, 2 \pi) \approx & \exp \left\{-i \pi g\left[|\psi(\theta, 0)|^{2}+|\psi(\theta+\pi, 0)|^{2}\right]\right\} \\
& \times\{\cos [F(\theta, 0)] \psi(\theta+\pi, 0)-\sin [F(\theta, 0)] \psi(\theta, 0)\}
\end{aligned}
$$

where we have defined $F(\theta, 0)=i \pi g\left[\psi^{*}(\theta, 0) \psi(\theta+\pi, 0)\right.$ $\left.-\psi(\theta, 0) \psi^{*}(\theta+\pi, 0)\right]$. Note that, in the limit $g \rightarrow 0$, Eqs. (6) and (7) become $\psi(\theta, 4 \pi)=\psi(\theta, 0)$ and $\psi(\theta, 2 \pi)=\psi(\theta+\pi, 0)$, as expected for the noninteracting free evolution. Using this approximation, we compute the evolution of the condensate for the first two kicks, starting from the initial condition $\psi(\theta, 0)=1 / \sqrt{2 \pi}$. We obtain

$$
\begin{aligned}
& \psi\left(\theta, 4 \pi^{+}\right) \approx \frac{1}{\sqrt{2 \pi}} \exp \left[-i V_{1}(\theta)\right] \exp (-i 2 g), \\
& \psi\left(\theta, 6 \pi^{+}\right) \approx \frac{1}{\sqrt{2 \pi}} \exp \left\{-i\left[V_{2}(\theta, \phi)-V_{1}(\theta)\right]\right\} \exp (-i 3 g) \\
& \times\left\{\cos \left[\Omega_{1}(\theta)\right]+\sin \left[\Omega_{1}(\theta)\right] \exp \left[-i 2 V_{1}(\theta)\right]\right\},
\end{aligned}
$$

where $\Omega_{1}(\theta)=g \sin \left(2 V_{1}(\theta)\right)$. The mechanism of the ratchet effect is now clear: due to atom-atom interactions, the modulus square of the wave function at time $6 \pi$ (before the second kick) is no longer constant in $\theta$. Instead we have, to first order in $g,|\psi(\theta, 6 \pi)|^{2} \approx \frac{1}{2 \pi}\left\{1+g \sin \left[4 V_{1}(\theta)\right]\right\}$, so that the initial constant probability distribution is modified by a term symmetric under the transformation $\theta \rightarrow-\theta$. The current after the kick at time $t=6 \pi$ is then given by

$$
\begin{aligned}
\left\langle p\left(6 \pi^{+}\right)\right\rangle & =-\int_{0}^{2 \pi} d \theta V_{2}^{\prime}(\theta, \phi)|\psi(\theta, 6 \pi)|^{2} \\
& \approx g k \int_{0}^{2 \pi} d \theta \sin (\theta-\phi) \sin (4 k \cos \theta) \\
& =-g k \sin (\phi) J_{1}(4 k),
\end{aligned}
$$

where $J_{1}$ is the Bessel function of the first kind of index 1 . This current is in general different from zero, provided that $V_{2}(\theta, \phi)$ is not itself symmetric under $\theta \rightarrow-\theta$, that is, when $\phi \neq 0, \pi$.

In Fig. 3, we show that it is possible to control the direction of transport by varying the phase $\phi$ : the current can be reversed simply by changing $\phi \rightarrow-\phi$. This current inversion can be explained by means of the following symmetry considerations. The evolution of the wave-function $\psi(\theta, t)$ is given by Eq. (1). After substituting in this equation $\theta \rightarrow-\theta$, and taking into account that $V(-\theta, \phi, t)=V(\theta,-\phi, t)$, we obtain

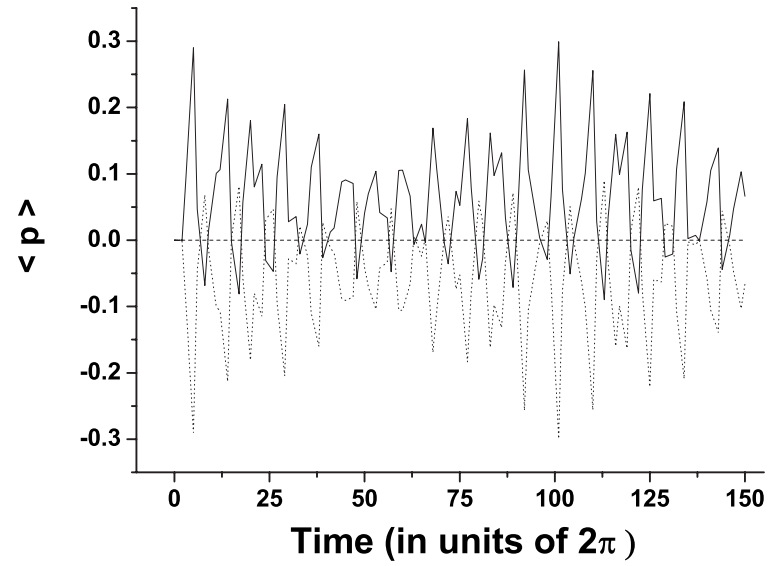

FIG. 3. Momentum vs time for different values of the parameter $\phi$, at $k \approx 0.74$ and $g=0.5: \phi=-\pi / 4$ (continuous curve), $\phi=0$ (dashed line), $\phi=\pi / 4$ (dotted curve).

$$
i \frac{\partial}{\partial t} \tilde{\psi}(\theta, t)=\left[-\frac{1}{2} \frac{\partial^{2}}{\partial \theta^{2}}+g|\tilde{\psi}(\theta, t)|^{2}+V(\theta,-\phi, t)\right] \tilde{\psi}(\theta, t),
$$

where $\widetilde{\psi}(\theta, t) \equiv \psi(-\theta, t)$. Therefore if $\psi(\theta, t)$ is a solution of the Gross-Pitaevskii equation, then also $\widetilde{\psi}(\theta, t)$ is a solution, provided that we substitute $\phi \rightarrow-\phi$ in the potential $V$. The momentum $\langle\tilde{p}(t)\rangle$ of the wave function $\tilde{\psi}(\theta, t)$ is obviously given by $\langle\tilde{p}(t)\rangle=-\langle p(t)\rangle$, where $\langle p(t)\rangle$ is the momentum of $\psi(\theta, t)$. This means that, for every $\psi(\theta, t)$ whose evolution is ruled by the Gross-Pitaevskii equation with potential $V(\theta, \phi, t)$, the wave function $\tilde{\psi}(\theta, t)$ evolves with exactly opposite momentum if $\phi \rightarrow-\phi$ in $V$. Since we start with an even wave function, $\tilde{\psi}(\theta, 0)=\psi(-\theta, 0)=\psi(\theta, 0)$, then changing $\phi \rightarrow-\phi$ changes the sign of the momentum of the wave function at any later time.

When studying the dynamics of a kicked BEC, it is important to take into account the proliferation of noncondensed atoms. Actually, strong kicks may lead to thermal excitations out of equilibrium and destroy the condensate, rendering the description by the Gross-Pitaevskii equation meaningless $[9,10]$. In the following, we show that, for the parameter values considered in this paper, the number of noncondensed particles is negligible compared to the number of condensed ones, thus demonstrating that our theoretical and numerical results based on the Gross-Pitaevskii equation are reliable.

Following the approach developed in [11] (see also [10]), we compute the mean number of noncondensed particles at zero temperature as $\delta N(t)=\sum_{j=1}^{\infty} \int_{0}^{2 \pi} d \theta\left|v_{j}(\theta, t)\right|^{2}$, where the evolution of $v_{j}(\theta, t)$ is determined by

$$
i \frac{\partial}{\partial t}\left[\begin{array}{l}
u_{j}(\theta, t) \\
v_{j}(\theta, t)
\end{array}\right]=\left[\begin{array}{cc}
H_{1}(\theta, t) & H_{2}(\theta, t) \\
-H_{2}^{*}(\theta, t) & -H_{1}^{\star}(\theta, t)
\end{array}\right]\left[\begin{array}{l}
u_{j}(\theta, t) \\
v_{j}(\theta, t)
\end{array}\right] .
$$

Here $\quad H_{1}(\theta, t)=H(\theta, t)-\mu(t)+g Q(t)|\psi(\theta, t)|^{2} Q(t), \quad H(\theta, t)$ $=-\frac{1}{2} \frac{\partial^{2}}{\partial \theta^{2}}+g|\psi(\theta, t)|^{2}+V(\theta, \phi, t)$ is the mean-field Hamiltonian 


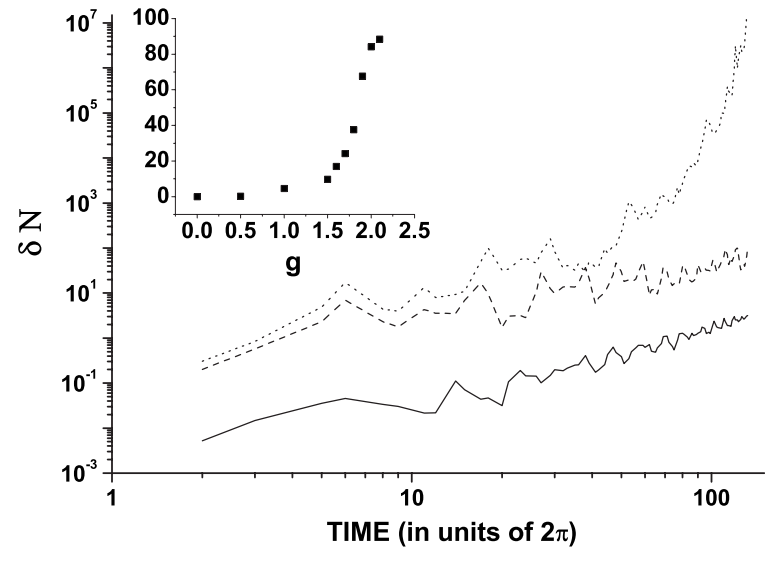

FIG. 4. Mean number $\delta N$ of noncondensed particles vs time for different values of the interaction strength $g$ : from bottom to top, $g=0.5,1.5$, and 2.0. Inset: $\delta N$ vs $g$ after 30 kicks. Parameter values: $k \approx 0.74, \phi=-\pi / 4$.

that governs the Gross-Pitaevskii Eq. (1), $\mu(t)$ is the chemical potential $[H(\theta, t) \psi(\theta, t)=\mu(t) \psi(\theta, t)], \quad Q(t)=1$ $-|\psi(t)\rangle\langle\psi(t)|$ projects orthogonally to $|\psi(t)\rangle$, and $H_{2}(\theta, t)$ $=g Q(t) \psi^{2}(\theta, t) Q^{*}(t)$.

We integrate in parallel Eqs. (1) and (11). The initial condition of the noncondensed part is obtained by diagonalizing the linear operator in Eq. (11) $[10,11]$. We obtain

$$
\left(\begin{array}{l}
u_{j}(\theta, 0) \\
v_{j}(\theta, 0)
\end{array}\right)=\frac{1}{2}\left(\begin{array}{l}
\xi+1 / \xi \\
\xi-1 / \xi
\end{array}\right) \frac{e^{i j \theta}}{\sqrt{2 \pi}}
$$

where $\xi=\left(\frac{j^{2} / 2}{j^{2} / 2+2 g|\psi(\theta, 0)|}\right)^{1 / 4}$, with $\psi(\theta, 0)=1 / \sqrt{2 \pi}$ initial condition of the BEC. The numerical evolution is performed using the split-operator method as in Eq. (5), with small integration steps $\tau \ll T$.

The number $\delta N$ of noncondensed particles, depending on the stability or instability of the condensate, grows polynomially or exponentially. As shown in Fig. $4, \delta N$ grows polynomially at small $g$ and exponentially for large $g$. The tran- sition from stability to instability takes place at $g=g_{c} \approx 1.7$. At $g>g_{c}$, thermal particles proliferate exponentially fast, $\delta N \sim \exp (r t)$, leading to a significant depletion of the condensate after a time $t_{d} \sim \ln (N) / r$. In this regime, the validity of the mean field description is undermined. On the other hand, for $g<g_{c}$ the exponential growth rate $r=0$ and the number of noncondensed particles is negligible for up to long times. For instance, as shown in Fig. $4, \delta N \approx 0.2(10)$ after $t=90 \pi$ (30 kicks) at $g=0.5(1.5)$, which is much smaller than the total number of particles $N \approx 10^{3}-10^{5}[10,12]$.

Finally, we would like to comment on the experimental feasibility of our proposal. The toruslike potential confining the BEC may be realized by using two two-dimensional circular optical billiards with the lateral dimension being confined by two plane optical billiards [13], where the circular barrier is obtained by deflecting a laser beam with acoustooptic deflectors fast enough so that this potential can be approximated by a static potential barrier. The toruslike potential may also be realized by using optical-dipole traps produced by red-detuned Laguerre-Gaussian laser beams of varying azimuthal mode index [14]. The kicks may be applied using a periodically pulsed strongly detuned laser beam transverse to the ring with the intensity linearly growing in one direction in the plane of the ring while constant in the other two directions, as proposed in [15]. The feasibility is also supported by the latest progress in the realization of BECs in optical traps such as the ${ }^{87} \mathrm{Rb}$ BEC in a quasi-onedimensional optical box trap, with condensate length $\sim 80 \mu \mathrm{m}$, transverse confinement $\sim 5 \mu \mathrm{m}$, and number of particles $N \sim 10^{3}$ [12]. Sequences of up to 25 kicks have been applied to a BEC of ${ }^{87} \mathrm{Rb}$ atoms confined in a static harmonic magnetic trap, with kicking strength $k \sim 1$ and in the quantum antiresonance case for the kicked oscillator model, $T=2 \pi$ [16]. Finally, the interaction strength $g$ can be tuned over a very large range using a Feshbach resonance or varying the number of atoms [17].

This work was supported in part by the MIUR COFIN-2005.
[1] R. D. Astumian and P. Hänggi, Phys. Today 55 (11), 33 (2002).

[2] P. Reimann, Phys. Rep. 361, 57 (2002).

[3] S. Flach, O. Yevtushenko, and Y. Zolotaryuk, Phys. Rev. Lett. 84, 2358 (2000).

[4] P. Reimann, M. Grifoni, and P. Hänggi, Phys. Rev. Lett. 79, 10 (1997); J. Lehmann, S. Kohler, P. Hänggi, and A. Nitzan, ibid. 88, 228305 (2002); G. G. Carlo, G. Benenti, G. Casati, and D. L. Shepelyansky, ibid. 94, 164101 (2005).

[5] H. Schanz, M. F. Otto, R. Ketzmerick, and T. Dittrich, Phys. Rev. Lett. 87, 070601 (2001); T. S. Monteiro, P. A. Dando, N. A. C. Hutchings, and M. R. Isherwood, ibid. 89, 194102 (2002); S. Denisov, L. Morales-Molina, and S. Flach, e-print arXiv:cond-mat/0607558; J. Gong, D. Polleti, and P. Hänggi, Phys. Rev. A 75, 033602 (2007).

[6] R. Gommers, S. Denisov, and F. Renzoni, Phys. Rev. Lett. 96,
240604 (2006) and references therein.

[7] G. G. Carlo, G. Benenti, G. Casati, S. Wimberger, O. Morsch, R. Mannella, and E. Arimondo, Phys. Rev. A 74, 033617 (2006).

[8] D. Poletti, L. Fu, J. Liu, and B. Li, Phys. Rev. E 73, 056203 (2006).

[9] S. A. Gardiner, D. Jaksch, R. Dum, J. I. Cirac, and P. Zoller, Phys. Rev. A 62, 023612 (2000).

[10] C. Zhang, J. Liu, M. G. Raizen, and Q. Niu, Phys. Rev. Lett. 92, 054101 (2004); J. Liu, C. Zhang, M. G. Raizen, and Q. Niu, Phys. Rev. A 73, 013601 (2006).

[11] Y. Castin and R. Dum, Phys. Rev. Lett. 79, 3553 (1997); Phys. Rev. A 57, 3008 (1998); Y. Castin, in Coherent Atomic Matter Waves, Les Houches Summer Schools, Session LXXII, edited by R. Kaiser, C. Westbrook, and F. David (Springer-Verlag, Berlin, 2001); C. W. Gardiner, Phys. Rev. A 56, 1414 (1997). 
[12] T. P. Meyrath, F. Schreck, J. L. Hanssen, C.-S. Chuu, and M. G. Raizen, Phys. Rev. A 71, 041604(R) (2005).

[13] V. Milner, J. L. Hanssen, W. C. Campbell, and M. G. Raizen, Phys. Rev. Lett. 86, 1514 (2001); N. Friedman, A. Kaplan, D. Carasso, and N. Davidson, ibid.. 86, 1518 (2001).

[14] E. M. Wright, J. Arlt, and K. Dholakia, Phys. Rev. A 63, 013608 (2000).
[15] B. Mieck and R. Graham, J. Phys. A 37, L581 (2004).

[16] G. J. Duffy, A. S. Mellish, K. J. Challis, and A. C. Wilson, Phys. Rev. A 70, 041602(R) (2004).

[17] E. A. Donley, N. R. Claussen, S. L. Cornish, J. L. Roberts, E. A. Cornell, and C. E. Wieman, Nature (London) 412, 295 (2001). 